\title{
El contrato de maternidad subrogada: La experiencia estadounidense*
}

\author{
Camilo A. Rodríguez-Yong** \\ Karol Ximena Martínez-Muñoz***
}

\begin{abstract}
RESUMEN
El artículo tiene como propósito identificar y presentar la experiencia de los Estados Unidos frente al contrato de maternidad subrogada. Esta experiencia tiene como características principales las siguientes: a) existe una regulación del contrato a partir de la ley y la jurisprudencia, b) no hay una posición uniforme entre los estados frente a la validez jurídica del contrato, c) se acude a la noción de orden público y a las normas del derecho de familia para determinar su validez, $y$ d) la regulación del contrato de maternidad subrogada está encaminada a contrarrestar los efectos negativos que se derivan del contrato. Igualmente, el artículo busca identificar los puntos comunes que existen entre esta visión y la postura colombiana en la materia.
\end{abstract}

Contrato de maternidad subrogada - alquiler de vientre - maternidad

\section{Surrogate motherbood contract. The US American experience}

\begin{abstract}
The article has the purpose of identifying and presenting the United States experience regarding the contract on surrogate motherhood. This experience has the following main features: a) there is a regulation of the contract founded in the law and case law, b) there is no uniform position among the states regarding the legal enforceability of the contract, c) public policy and family law are used to determine the legal validity of the agreement, and d) the regulation of the contract is directed to counter the negative effects of contract. Likewise, the article wants to identify the common points between the U.S's vision and the Colombian.
\end{abstract}

Surrogate motherhood contract - womb for hire - motherhood

* Este artículo se realizó dentro de la línea de investigación de derecho civil del grupo de derecho privado de la Facultad de Jurisprudencia de la Universidad del Rosario, Colombia.

** Abogado, Máster en Derecho, Director del grupo de investigación en derecho privado, investigador y profesor, Facultad de Jurisprudencia de la Universidad del Rosario, Colombia. Camilo.rodriguez@urosario. edu.co

*** Abogada, Máster en Derecho, Directora de Pregrado en la Facultad de Jurisprudencia de la Universidad del Rosario, Colombia. Karol.martinez@urosario.edu.co

Artículo recibido el 5 de junio de 2012 y aceptado para su publicación por el Comité Editorial el 26 de octubre de 2012. 


\section{INTRODUCCIÓN}

$\mathrm{E}$ 1 desarrollo de la ciencia y la tecnología ha impactado de manera clara y profunda en todos los aspectos de la vida en sociedad. Sin duda alguna, los nuevos descubrimientos e inventos han traído como consecuencia que los seres humanos vivamos hoy en una forma muy distinta a la que lo hacíamos hace algunas décadas. En el campo médico, los avances científicos han sido particularmente importantes y significativos. Allí, año tras año, la sociedad ha sido testigo de la aparición de nuevas vacunas, procedimientos o instrumentos científicos encaminados a la cura o tratamiento de enfermedades físicas o sicológicas. La búsqueda de soluciones médicas al problema de la infertilidad de hombres y mujeres representa uno de los campos en donde se han presentado grandes avances, siendo uno de ellos los tratamientos de reproducción asistida.

Desde este contexto, emergió la figura de la subrogación, la cual puede dividirse en tradicional y gestacional ${ }^{1}$. La primera se presenta cuando una mujer acepta quedar embarazada con la esperma de un hombre casado, y luego, una vez que se produzca el nacimiento del bebé fruto de ese procedimiento, se entiende que los padres del recién nacido son el hombre y su esposa. Por su parte, la subrogación gestacional existe cuando la esperma de un hombre casado se integra con el ovocito o huevo de su esposa, y el embrión que resulta de dicho procedimiento se implanta posteriormente en el útero de otra mujer ${ }^{2}$. Estas modalidades también se conocen con el nombre de subrogación parcial y subrogación total ${ }^{3}$.

La subrogación permitió la aparición de la figura de la maternidad subrogada. Esta ha sido considerada por muchos como un procedimiento controversial debido a los efectos negativos que produce para las madres subrogadas, es decir, aquellas que aceptan quedar embarazadas y gestar un bebé en su cuerpo. Por ejemplo, se ha dicho que la renuncia de la madre subrogada al recién nacido constituye un acto muy doloroso para ella, el cual puede dar lugar a la aparición de problemas sicológicos. Igualmente,

\footnotetext{
${ }^{1}$ Sin embargo, los orígenes de la maternidad subrogada no se remontan al siglo XX, pues algunos han considerado que ya desde épocas ancestrales era posible identificar un fenómeno similar. De acuerdo con el antiguo testamento, Sarah, esposa de Abraham, y quien no podía tener hijos, convenció a este último para que engendrara un hijo con su empleada Agar, para que de esta manera Sara pudiera ser madre. El bebé nacido de Abraham y Agar, el cual fue llamado Ismael, fue criado como hijo de Sara y Abraham. Behm, L. L., "Legal, Moral \& International Perspectives on Surrogate Motherhood: the Call for a Uniform Regulatory Scheme in the United States", en DePaul Journal of Health Care Law, DePaul University, Vol. 3, Núm. 2, 1999, p. 560. Igualmente, en el caso particular de los Estados Unidos, la doctrina ha identificado el fenómeno de las madres subrogadas durante la época anterior a la guerra civil norteamericana. En aquella época las mujeres esclavas gestaban bebés que no les pertenecerían a ellas como madres biológicas, sino a sus amos. Spivack, C., "The Law of Surrogate Motherhood in the United States", en The American Journal of Comparative Law, American Society of Comparative Law, Núm. 58, Suplemento, 2010, pp. 97-98.

${ }^{2}$ Cynthia v. Moschetta, 25 Cal. App. 4th 1218 (1994).

3 Jadva, V., Murray, C., Lycett, E., Maccallum, F. y Golombok, S., "Surrogacy: the Experiences of Surrogate Mothers", en Human Reproduction, Oxford University Press, Vol. 10, Núm. 18, 2003, p. 2196. Ver también Van Den Akker, O., "Psychosocial aspects of surrogate motherhood", en Human Reproduction Update, Oxford University Press, Vol. 1, Núm. 13, 2007, p. 53.
} 
se ha señalado que la maternidad subrogada puede poner en riesgo la salud de la madre gestante y el bebé, pues aquella puede distanciarse de este al considerar que no es suyo o no le pertenece. Finalmente, se ha afirmado que luego de producida la separación de la madre subrogada del recién nacido, esta puede desarrollar sentimientos de depresión postparto, rabia o culpa ${ }^{4}$.

A pesar de los efectos negativos de los cuales ha sido acusada la práctica de la maternidad subrogada, existen varias razones por las cuales las mujeres deciden hacer parte de este procedimiento. En el año 2003 se realizó una encuesta a 34 mujeres que habían sido madres subrogadas. Allí se pidió a estas que identificaran las razones que las motivaron a participar, obteniéndose los siguientes resultados. El 91\% (31 personas) de ellas manifestó que había decidido participar en la subrogación porque quería ayudar a la pareja que no tenía hijos, el $15 \%$ ( 5 personas) porque disfrutaba del embarazo, el $6 \%$ (2 personas) por autorrealización (self-fulfillment), y finalmente, $3 \%$ ( 1 persona) por el pago de una remuneración 5 .

Por su parte, las parejas que han decidido tener un hijo mediante la figura de la maternidad subrogada han tenido como motivaciones principales la infertilidad de la pareja, el tiempo y los costos inherentes a un proceso de adopción, la existencia de una anormalidad genética en la esposa que puede ser transmitida al bebé, y la decisión de

${ }^{4}$ Ibid., "Sin embargo, algunos estudios han concluido que no hay una evidencia clara y concluyente sobre la real ocurrencia de estos efectos. A further source of unease in relation to surrogacy is the possibility of adverse psychological consequences for the surrogate mother. Although the study showed that surrogate mothers did experience some problems immediately after the handover, these were not severe, tended to be short-lived, and to dissipate with time. One year on, only two women -both of whom were known surrogate mothers-reported feeling occasionally upset. Furthermore, the Edinburgh Depression Scale showed that none of the surrogate mothers was suffering from post-natal depression at 1 year following the birth. Thus, there was no evidence of difficulties with respect to those aspects of surrogacy that have been the greatest cause for concern. Overall, surrogacy appears to be a positive experience for surrogate mothers. Women who decide to embark upon surrogacy often have completed a family of their own and feel they wish to belp a couple who would not otherwise be able to become parents. The present study lends little support to the commonly held expectation that surrogate mothers will experience psychological problems following the birth of the child. Instead, surrogate mothers often reported a feeling of self-worth. Ibid., p. 2203. ["Una fuente adicional de inquietud en relación con la subrogación es la posibilidad de consecuencias sicológicas adversas para la madre subrogada. Aunque el estudio mostró que las madres subrogadas si experimentaron algunos problemas inmediatamente después de la entrega, estos no fueron severos, tendieron a ser cortos y a disiparse con el tiempo. Un año después, solo dos mujeres -ambas madres subrogadas- reportaron sentirse ocasionalmente alteradas. Además, la escala de depresión de Edimburgo mostró que ninguna de las madres subrogadas estaba sufriendo de depresión posparto un año después del nacimiento. Por lo tanto, no hay evidencia de dificultades respecto de aquellos aspectos de la subrogación que han sido la mayor fuente de preocupación. En general, la subrogación parece ser una experiencia positiva para las madres subrogadas. Las mujeres que deciden embarcarse en la subrogación generalmente han tenido su propia familia y sienten que ellas desean ayudar a parejas que no de otro modo no podrían ser padres. El presente estudio presta poco apoyo a la expectativa común de que las madres subrogadas experimentarán problemas sicológicos luego del nacimiento del recién nacido. Por el contrario, las madres subrogadas con frecuencia reportaron un sentimiento de autoestima"].

${ }^{5}$ Las encuestadas podían seleccionar varias respuestas. Ibíd., p. 2199. 
la mujer, por razones de conveniencia y sin que exista una razón médica, de no tener un hijo por ella misma ${ }^{6}$.

El fenómeno de la maternidad subrogada ha tenido un gran desarrollo en países como Estados Unidos, en donde, por medio de normas y decisiones judiciales, se ha ido regulando su ejercicio, aunque no de manera uniforme. En consideración a lo anterior, este artículo tiene como propósito central identificar las características o principios fundamentales que conforman la visión de los Estados Unidos en materia de los contratos de maternidad subrogada. La determinación de estas características es el resultado de la revisión y análisis de las distintas normas y pronunciamientos judiciales proferidos por las cortes estadounidenses.

Bajo este contexto, los rasgos distintivos que han sido identificados como característicos de la postura estadounidense son los siguientes: a) existe una regulación del contrato a partir de la ley y la jurisprudencia, b) no hay una posición uniforme entre los estados frente a la validez jurídica del contrato, c) se acude a la noción de orden público y a las normas del derecho de familia para aceptar o rechazar su validez, y d) la regulación del contrato está encaminada a contrarrestar los efectos negativos que se derivan del fenómeno de maternidad subrogada.

La identificación de la aproximación estadounidense a los contratos de maternidad subrogada es de gran importancia y relevancia, toda vez que puede servir a países que no tienen una regulación sobre esta materia, y por lo tanto pueden encontrar en esta experiencia herramientas útiles que le permitan reglarlo de una mejor manera.

De otra parte, y aprovechando la identificación de los rasgos distintivos que caracterizan la visión estadounidense frente a los contratos de maternidad subrogada, este artículo tiene un segundo propósito, cual es, identificar los puntos comunes que existen entre esta visión y la postura colombiana que puede construirse a partir de la jurisprudencia de la Corte Constitucional y los diversos intentos regulatorios que se han presentado, esto es, los proyectos de Ley No 46 de 2003-Senado, No 196 de 2008 - Cámara de Representantes y No 37 de 2009. Recuérdese que en Colombia no existe una regulación especial vigente sobre los contratos de maternidad subrogada, a pesar de que la Corte Constitucional ha reconocido la necesidad de tenerla ${ }^{7}$ y la ocurrencia de varios intentos legislativos fallidos.

${ }^{6}$ Tiller, S. L., "Litigation, Legislation, and Limelight: Obstacles to Commercial Surrogate Mother Arrangements”, en Iowa Law Review, Iowa University, Vol. 2, Núm. 72, 1987, p. 418.

${ }^{7}$ El llamado a la regulación del contrato encuentra justificación en el reconocimiento de que el fenómeno de maternidad subrogada no es ajeno a la sociedad colombiana. "En Colombia, al parecer también es una práctica en auge. En internet se encuentran cientos de anuncios de mujeres de todas las edades que ofrecen su vientre para hacer realidad el sueño de otros de ser padres”. Corte Constitucional T-968/09, M.P. María Victoria Calle, 18/12/2009. En efecto, en páginas de internet locales es posible encontrar anuncios de personas que buscan celebrar un contrato de este tipo. “¿Quieres Ser MADRE SUBROGADA (Sustituta)? Preferentemente que viva en Medellín o sus alrededores. Obligatoriamente mayor de edad, sin antecedentes delictivos y con disponibilidad de atender el proceso legal en juzgados. No respondas si no puedes ser contactada telefónicamente o no tienes disponibilidad de fotos REALES, serias, de cara y cuerpo. Sólo tendremos en cuenta, para contactar, las que tengan: - CIUDAD DE RESIDENCIA - NOMBRE 
Conocer las similitudes existentes entre la visión estadounidense de los contratos de maternidad subrogada y lo hasta ahora planteado en el contexto colombiano, permite conocer qué tan cerca o lejanas están una de la otra, y en consecuencia, valorar de manera más eficaz la posibilidad de recurrir a la experiencia norteamericana para la solución de conflictos sobre la materia o la regulación del contrato en el país.

\section{Definición del Contrato de Madre Subrogada}

El contrato de madre subrogada ha sido definido como un acuerdo por medio del cual una mujer acepta quedar embarazada mediante un procedimiento de inseminación artificial, para que luego, una vez que se produzca el nacimiento del bebé, lo entregue al donante de la esperma y su esposa, renunciando para ello a los derechos que la ley le confiere sobre el recién nacido, y en contraprestación, por regla general, al pago de una compensación, generalmente consistente en una suma de dinero ${ }^{8}$.

Una definición en esta dirección ha sido adoptada por la legislación de algunos estados de la unión americana. Por ejemplo, la ley del estado de Luisiana concibe el contrato de madre subrogada bajo los siguientes términos:

Contract for surrogate motherhood" means any agreement whereby a person not married to the contributor of the sperm agrees for valuable consideration to be inseminated, to carry any resulting fetus to birth, and then to relinquish to the contributor of the sperm the custody and all rights and obligations to the child. [Un contrato de maternidad subrogada significa un acuerdo por medio del cual una persona no casada con el contribuyente de la esperma acuerda por una contraprestación de valor ser inseminada, llevar el

COMPLETO - CORREO ELECTRÓNICO - TELÉFONO DE CONTACTO. Favor NO enviar otros datos por este medio. CUIDAMOS TU PRIVACIDAD, NO ENTREGAMOS TUS DATOS NI FOTOS. PARA CONTACTARTE NO DEJAMOS MENSAJES, NI HABLAMOS CON TERCEROS (NOVIOS, PAREJAS, ETC.). La entrevista para información únicamente es con cita en un consultorio o en oficina (horas hábiles), con la profesional a cargo, que es quien hace la llamada de contacto. Somos muy respetuosos, por lo que no creamos falsas expectativas, ofreciendo altas sumas de dinero o casas, que a la postre solo son especulaciones. Ni tampoco garantizamos que sea la escogida por los interesados. Gracias". http://antioquia.evisos.net/ comunidad/anuncios-personales/quieres-ser-madre-subrogada-sustituta.html. En otro anuncio se dijo lo siguiente:"mi esposo y yo hemos decidido buscar un vientre de alquiler y poder realizar el sueño de ser padres. Si tú vives en Cali y crees que puedes ayudarnos por favor no dudes en cominicarte (sic) con nosotros. Detrás de cada caso hay personas que necesitan de ayuda y hoy necesitamos la tuya. http://foro.enfemenino. com/forum/f392/_f144_f392-Buscamos-vientre-de-alquiler-en-cali-colombia.html

${ }^{8}$ Gallee, C. L., "Surrogate Mother Contracts: A View of Recent Legislative Approaches", en Journal of Health Law, American Health Law Associations, Vol. 6, Núm. 24, 1992, pp. 175-176; Cohen, B., "Surrogate Mothers: Whose Baby Is It?", en American Journal of Law E Medicine, Boston University School of Law, Vol. 3, Núm. 10, 1984, p. 243. 
feto resultante hasta el nacimiento, y luego entregar al contribuyente de la esperma la custodia y todos los derechos y obligaciones del recién nacido] $]^{9}$.

Por su parte, el estado de Michigan definió el contrato así:

Surrogate parentage contract" means a contract, agreement, or arrangement in which a female agrees to conceive a child through natural or artificial insemination, or in which a female agrees to surrogate gestation, and to voluntarily relinquish her parental or custodial rights to the child. It is presumed that a contract, agreement, or arrangement in which a female agrees to conceive a child through natural or artificial insemination by a person other than her husband, or in which a female agrees to surrogate gestation, includes a provision, whether or not express, that the female will relinquish her parental or custodial rights to the child. [Un "contrato de ascendencia subrogada" significa un contrato, acuerdo o arreglo en el cual una mujer acuerda concebir un niño mediante inseminación natural o artificial, o en el cual una mujer acuerda sustituir en la gestación, y voluntariamente renunciar a sus derechos parentales o de custodia sobre el niño. Se presume que en un contrato, acuerdo o arreglo en el cual la mujer acuerda concebir un niño por medio de inseminación natural o artificial por una persona diferente a su esposo, o en el cual la mujer acuerda sustituir en la gestación, incluye una provisión, expresa o no, de que la mujer renunciará a sus derechos parentales o de custodia sobre el niño] ${ }^{10}$.

El contrato de maternidad subrogada puede clasificarse en altruista y comercial. El primero se presenta cuando la madre no recibe pago alguno por el alquiler de su vientre, o si recibe alguna contraprestación, esta se limita al pago de los gastos derivados del embarazo. Por el contrario, el contrato de maternidad subrogada de carácter comercial existe cuando la madre sí recibe un pago como contraprestación de las obligaciones derivadas del contrato $^{11}$, pago que puede consistir, no solamente en una suma de dinero, sino también en la entrega de objetos, servicios o cualquier otra cosa de valor pecuniario ${ }^{12}$.

A partir de las anteriores consideraciones, es posible identificar un conjunto de obligaciones particulares que surgen para las partes del contrato. Para el caso de la madre subrogada, estas consistirán en: 1) permitir ser inseminada artificialmente con la esperma del padre biológico, 2) llevar el feto en su vientre hasta el nacimiento del

${ }^{9}$ Otros estados que han definido legislativamente el contrato han sido New Hampshire (New Hampshire Code 168-B:1 y ss), Virginia (Code of Virginia§ 20-156 y ss), Washington (Revised Code of Washington $\S 26.26 .210$ y ss), el Distrito de Columbia (District of Columbia Code $§ 16-401$ y ss) y Nueva York (NY CLS Dom Rel $\$ 121$ y ss), entre otros.

${ }^{10}$ MCL $\$ 722.853$.

${ }^{11}$ McLachlan, H. V. y Swales, J. K., "Show Me the Money: Making Markets in Forbidden Exchange: Commercial Surrogate Motherhood and the Alleged Commodification of Children: a Defense of Legally Enforceable Contracts", en Law and Contemporary Problems, Duke University, Vol. 3, Núm. 72 2009, p. 92.

${ }^{12}$ Revised Code of Washington $\$ 26.26 .210$. 
bebé, y 3) renunciar a los derechos de custodia sobre el recién nacido en favor del padre biológico y su esposa.

Por su parte, en contraprestación a las obligaciones asumidas por la madre subrogada, el padre biológico y su esposa se obligan a: 1) pagar todos los gastos médicos y legales generados como consecuencia del embarazo, 2) asumir la responsabilidad de custodia sobre el recién nacido, y 3) como regla general, pagar a la madre subrogada una compensación ${ }^{13}$.

\section{LA VISIÓN ESTADOUNIDENSE SOBRE EL CONTRATO DE MATERNIDAD SUBROGADA}

La sociedad de los Estados Unidos no ha sido ajena al fenómeno de la maternidad subrogada. De acuerdo con estadísticas de la Society for Assisted Reproductive Technology (SART), el número de bebés nacidos de madres subrogadas aumentó de manera sustancial en el período de 2004 a 2008, pasando de 738 a casi $1.400^{14}$. Igualmente, el fenómeno de la maternidad subrogada ha impactado también en el campo judicial y legislativo del país norteamericano, escenarios en donde se ha discutido acerca de la validez de los contratos de maternidad subrogada.

Precisamente, a partir de la revisión y análisis de las leyes y decisiones judiciales estadounidenses, es posible establecer e identificar las características que constituyen la visión o aproximación norteamericana frente a estos contratos, características que son presentadas a continuación.

\subsection{La regulación del contrato de maternidad subrogada se ha construido a partir de la ley y la jurisprudencia}

El primer rasgo característico de la experiencia norteamericana frente a los contratos de maternidad subrogada es que estos se encuentran regulados por una serie de reglas y principios jurídicos que tienen origen legislativo y judicial. Lo anterior, debido a que algunos estados de la unión americana han promulgado leyes particulares o especiales sobre la materia, mientras que otros se han abstenido de hacerlo, dejando en este último caso la regulación del contrato a la jurisprudencia, es decir, a las decisiones de los jueces.

En el caso de las legislaciones estatales en materia de contratos de maternidad subrogada, estas fueron promulgadas con el propósito de, por un lado, establecer de manera clara los derechos de custodia del recién nacido ${ }^{15}$, y por el otro, proteger a las

\footnotetext{
13 Tiller, S. L. Op. cit., pp. 417-418.

${ }^{14}$ Gugucheva, M., Surrogacy in America, Council for Responsible Genetics, 2010, p. 4, disponible en http://www.councilforresponsiblegenetics.org/pageDocuments/KAEVEJ0A1M.pdf (última visita 01/03/12).

${ }^{15}$ Por ejemplo, la ley de Arizona establece: B. A surrogate is the legal mother of a child born as a result of a surrogate parentage contract and is entitled to custody of that child. [B. Una madre subrogada es la madre legal de un niño nacido como resultado de un contrato de maternidad subrogada y tiene derecho a la custodia de
} 
partes del contrato por el establecimiento de estándares y salvaguardas que respetarán el orden público del estado ${ }^{16}$.

Igualmente, la jurisprudencia también ha sido una fuente fundamental en la construcción del análisis jurídico de los contratos de maternidad subrogada. Sin embargo, debe destacarse que la propia jurisprudencia ha resaltado que el mejor escenario para construir una aproximación jurídica al fenómeno de maternidad subrogada es mediante el poder legislativo. En esta dirección se pronunció la Suprema Corte de California en el caso Johnson v. Calvert, al señalar:

We are all too aware that the proper forum for resolution of this issue is the Legislature, where empirical data, largely lacking from this record, can be studied and rules of general applicability developed. [Nosotros somos conscientes de que el foro adecuado para resolución de este asunto es el legislativo, en donde datos empíricos, que faltan ampliamente en este caso, pueden ser estudiados y reglas de aplicación general desarrolladas] ${ }^{17}$.

Esta primera característica de la experiencia norteamericana difiere a lo acontecido en el caso colombiano, pues, como se mencionó anteriormente, en Colombia no existe actualmente una regulación particular sobre el contrato de maternidad subrogada. No obstante, sí se han presentado varios intentos legislativos, aunque fallidos, por regularlo. Precisamente, la ausencia de regulación, sumada al reconocimiento de la ocurrencia del fenómeno de la maternidad subrogada en el país, motivó la presentación de diversos proyectos de ley. Así por ejemplo, fue reconocido en la exposición de motivos del proyecto de ley Nº37 de 2009 de la Cámara de Representantes colombiana. Allí se manifestó: "No podemos estar ajenos al vacío jurídico que en la actualidad se encuentra en nuestro ordenamiento; la práctica reiterada de esta figura en nuestro país hace necesaria la urgente actividad del legislador para reglamentar una situación que no puede ser ajena a la evolución de la procreación de la humanidad"18.

ese niño]. Arizona Revised Statues $§ 25-218$. Igualmente, la ley de Dakota señala: Any agreement in which a woman agrees to become a surrogate or to relinquish that woman's rights and duties as parent of a child conceived through assisted conception is void. The surrogate, however, is the mother of a resulting child and the surrogate's husband, if a party to the agreement, is the father of the child. If the surrogate's husband is not a party to the agreement or the surrogate is unmarried, paternity of the child is governed by chapter 14-20. [Cualquier acuerdo en el cual una mujer conviene convertirse en una madre subrogada o renunciar a su derecho y deberes como madre de un niño concebido mediante una concepción asistida es nulo. La madre subrogada, sin embargo, es la madre del niño resultante y su esposo, si es parte del acuerdo, es el padre del niño. Si el esposo de la subrogada no es parte del contrato o la subrogada no está casada, la paternidad del niño estará regulada por el capitulo 14-20]. North Dakota Code $\$ 14-18-05$.

${ }^{16}$ Illinois, 750 ILCS 47/5. Por ejemplo, en el estado de Utah, la norma estatal tuvo como intención particular la protección a las madres subrogadas. J.R. v. The State of Utah, 261 F. Supp. 2d 1268 (2003).

17 Johnson v. Calvert, 5 Cal. 4th 84 (1993).

18 Exposición de motives Proyecto de Ley 037 Cámara de Representantes. Igual se manifestó en el proyecto de ley 196 de 2008. 
De otra parte, desde la perspectiva jurisprudencial, es importante resaltar la sentencia T-968 de 2009 proferida por la Corte Constitucional colombiana, pues allí, a pesar de que no se estableció una regulación al contrato de maternidad subrogada (algo que por supuesto no podía hacer la Corte), sí señaló dos aspectos fundamentales. Primero, reconoció la validez de este tipo de contratos en Colombia al no existir norma expresa que los prohíba. Segundo, resaltó la necesidad de que haya una "regulación exhaustiva" y el establecimiento de una serie de requisitos o condiciones para la celebración del contrato $^{19}$. Frente a esta necesidad exaltada por la Corte Constitucional, toma fundamental importancia revisar y conocer experiencias foráneas sobre la materia, pues de ellas podrán identificarse aspectos y experiencias positivas que permitan la adopción de una adecuada regulación en el territorio colombiano.

\subsection{No existe una posición uniforme entre los estados de la unión americana frente a la validez jurídica de los contratos de maternidad subrogada}

Un segundo rasgo distintivo de la experiencia norteamericana en relación con los contratos de maternidad subrogada, es la falta de una postura uniforme frente a su validez o invalidez jurídica ${ }^{20}$. En efecto, mientras un grupo de estados rechaza de manera expresa

${ }^{19}$ Corte Constitucional T-968/09, M.P. María Victoria Calle, 18/12/2009.

20 "A significant minority of States have legislation addressing surrogacy agreements. Some simply deny enforcement of all such agreements. See Ariz. Rev. Stat. Ann. § 25-218(A) (West 1991); D.C. Code Ann. § 16-402(a) (1997); Ind. Code Ann. § § 31-20-1-1, 31-20-1-2 (Michie 1997); Mich. Comp. Laws Ann. $\S 722.855$ (West 1993); N.Y. Dom. Rel. Law $\$ 122$ (McKinney Supp. 1997), N.D. Cent. Code $\$ 14-18-05$ (1991); Utah Code Ann. § 76-7-204 (1995). Others expressly deny enforcement only if the surrogate is to be compensated. See Ky. Rev. Stat. Ann. § 199.590(4) (Michie 1995); La. Rev. Stat. Ann. § 9:2713 (West 1991); Neb. Rev. Stat. § 25-21,200 (1995); Wash. Rev. Code $§ ~ 26.26 .230,26.26 .240$ (1996). Some States have simply exempted surrogacy agreements from provisions making it a crime to sell babies. See Ala. Code § 26-10A-34 (1992); Iowa Code § 710.11 (1997); W. Va. Code § 48-4-16(e)(3) (1996). A few States have explicitly made unpaid surrogacy agreements lawful. See Fla. Stat. ch. 742.15 (1995); Nev. Rev. Stat. § 126.045 (1995); N.H. Rev. Stat. Ann. § 168-B:16 (1994 \& Supp. 1996); Va. Code Ann. §§ 20-159, 20-160(B)(4) (Michie 1995). Florida, New Hampshire, and Virginia require that the intended mother be infertile. See Fla. Stat. ch. 742.15(2)(a); N.H. Rev. Stat. Ann. § 168-B:17(II) (1994); Va. Code Ann. § 20160(B)(8). New Hampshire and Virginia place restrictions on who may act as a surrogate and require advance judicial approval of the agreement. See N.H. Rev. Stat. Ann. $\S 168-B: 16(I)(b), 168-B: 17$; Va. Code Ann. $\S \S 20-159$ (B), 20-160(B)(6). Last, Arkansas raises a presumption that a child born to a surrogate mother is the child of the intended parents and not the surrogate. Ark. Code Ann. § 9-10-201(b),(c) (Michie 1993)”. [Una minoría significativa de estados tiene legislación sobre acuerdos de maternidad subrogada. Algunos simplemente niegan validez a todos esos acuerdos. Vea Ariz. Rev. Stat. Ann. § 25-218(A) (West 1991); D.C. Code Ann. § 16-402(a) (1997); Ind. Code Ann. § § 31-20-1-1, 31-20-1-2 (Michie 1997); Mich. Comp. Laws Ann. $\$ 722.855$ (West 1993); N.Y. Dom. Rel. Law $\$ 122$ (McKinney Supp. 1997), N.D. Cent. Code § 14-18-05 (1991); Utah Code Ann. § 76-7-204 (1995). Otros expresamente les niegan validez solamente si la madre subrogada es compensada. Vea Ky. Rev. Stat. Ann. § 199.590(4) (Michie 1995); La. Rev. Stat. Ann. § 9:2713 (West 1991); Neb. Rev. Stat. § 25-21,200 (1995); Wash. Rev. Code $§ \S 26.26 .230,26.26 .240$ (1996). Algunos otros estados han simplemente excluido a los acuerdos de maternidad subrogada de las normas que consideran la venta de bebés un crimen. Vea Ala. Code $\$ 26-10 A-34$ (1992); Iowa Code $\$ 710.11$ (1997); W. Va. Code § 48-4-16(e)(3) (1996). Unos pocos estados han expresamente calificado como válidos los acuerdos de maternidad subrogada gratuitos. Vea Fla. Stat. ch. 742.15 (1995); Nev. Rev. Stat. § 126.045 
y clara la celebración de estos contratos, tal como ocurre en los de Arizona, Indiana, Nueva York y el Distrito de Columbia, otro grupo, por el contrario, le reconoce plena validez, aunque en algunos casos, siempre y cuando se cumpla con un procedimiento especial establecido por la ley ${ }^{21}$. Entre los estados que han adoptado esta última postura se encuentran los de New Hampshire ${ }^{22}$, Florida ${ }^{23}$, Illinois ${ }^{24}, \operatorname{Utah}^{25}$ y Virginia ${ }^{26}$.

En lo que respecta a Colombia, a pesar de que no existe una regulación específica aplicable al fenómeno de la maternidad subrogada, la Corte Constitucional ha reconocido que esta práctica no se encuentra expresamente prohibida. Inclusive, puede encontrarse su justificación o legitimación en el artículo 42 de la Constitución Política. Este artículo señala: "Los hijos habidos en el matrimonio o fuera de él, adoptados o procreados naturalmente o con asistencia científica, tienen iguales derechos y deberes" ${ }^{27}$.

Sin embargo, es importante destacar que en los diferentes proyectos de ley que buscaron regularla, una primera postura se oponía a su reconocimiento. En efecto, el artículo $9^{\circ}$ del proyecto de ley 46 de 2003 calificaba expresamente como nulo un contrato de alquiler de útero. Adicionalmente, el artículo 16 del mismo proyecto establecía la misma sanción para "cualquier contrato por el que se convenga la gestación, a cargo de una mujer que renuncia a la filiación materna a favor del contratante o de un tercero".

Sin embargo, de manera posterior otros proyectos, como el 37 de 2009 o el 196 de 2008, sí autorizaron la celebración de contrato de maternidad subrogada, aunque limitando o condicionando su utilización a la falta de capacidad reproductiva de uno de los miembros de la pareja solicitante o interesada en la realización del procedimiento. En

(1995); N.H. Rev. Stat. Ann. § 168-B:16 (1994 \& Supp. 1996); Va. Code Ann. §§ 20-159, 20-160(B)(4) (Michie 1995). La Florida, New Hampshire y Virginia requieren que la madre interesada sea infértil. Vea Fla. Stat. ch. 742.15(2)(a); N.H. Rev. Stat. Ann. § 168-B:17(II) (1994); Va. Code Ann. § 20-160(B)(8). New Hampshire y Virginia establecen restricciones en quien puede actuar como madre subrogada y solicita una aprobación judicial anticipada del contrato. Vea N.H. Rev. Stat. Ann. §§ 168-B:16(I)(b), 168-B:17; Va. Code Ann. §§ 20-159(B), 20-160(B)(6). Por último, Arkansas establece una presunción de que el recién nacido de una madre subrogada es hijo de los padres interesados y no de la madre subrogada. Ark. Code Ann. § 9-10-201(b),(c) (Michie 1993)”. R.R. v. M.H, 426 Mass. 501 (1998)]. R.R. v. M.H, 426 Mass. 501 (1998).

${ }^{21}$ A surrogate arrangement is lawful only if it conforms to the requirements of this subdivision... [Un contrato de maternidad subrogada es legal solamente si cumple con los requisitos de esta subdivisión] New Hampshire Code 168-B:16.

22 Ibid.

${ }^{23}$ Florida Statute $\$ 742.15$.

${ }^{24}$ Illinois Compiled Statutes 750 ILCS 47/20.

${ }^{25}$ Utah Code 78B-15-801.

${ }^{26}$ Code of Virginia $\$ 20-159$.

27 "En el ordenamiento jurídico colombiano no existe una prohibición expresa para la realización de este tipo de convenios o acuerdos. Sin embargo, respecto de las técnicas de reproducción asistida, dentro de las cuales se ubica la maternidad subrogada o sustituta, la doctrina ha considerado que están legitimadas jurídicamente, en virtud del artículo 42-6 constitucional...". Corte Constitucional T-968/09, M.P. María Victoria Calle, 18/12/2009. Gustavo Adolfo Marín Vélez, El arrendamiento de vientre en Colombia, Opinión Jurídica 69-85, Sello editorial Universidad de Medellín, Vol. 3, 2003, p. 71. 
otras palabras, solamente en aquellos casos en donde uno de los miembros de la pareja no tuviera la capacidad de tener hijos, podía celebrarse el contrato ${ }^{28}$.

En consecuencia, sobre las anteriores consideraciones, es posible afirmar que tanto en los Estados Unidos (para el caso de algunos estados) como en Colombia el contrato de maternidad subrogada ha sido aceptado como un contrato válido. No obstante, también en ambos casos, es posible identificar visiones contrarias que buscan negarle cualquier efecto jurídico. Sin embargo, mientras que en el caso colombiano esta última postura no tiene fuerza jurídica pues está restringida a un proyecto de ley, en el caso estadounidense sí la tiene plenamente reconocida en varios estados.

\subsection{La noción de orden público es el criterio alrededor del cual gira el análisis de la validez jurídica del contrato}

La tercera característica de la experiencia norteamericana con relación al contrato de maternidad subrogada, es la utilización del concepto de orden público como criterio fundamental para determinar su validez jurídica. En efecto, como acaba de mencionarse, existen dentro de los Estados Unidos posiciones disímiles frente a la validez jurídica de los contratos de maternidad subrogada. Al revisar la jurisprudencia y la legislación norteamericana sobre la materia, se puede concluir que la adopción de una postura positiva o negativa frente a los mencionados contratos, encuentra sustento en el concepto o noción de orden público que pretende proteger el respectivo estado.

Por ejemplo, la Suprema Corte de Ohio ha señalado que los contratos de maternidad subrogada no son violatorios del orden público, y por tanto, deben honrarse las obligaciones y derechos que se asumen mediante estos: A written contract defining the rights and obligations of the parties seems an appropriate way to enter into surrogacy agreement. If the parties understand their contract rights, requiring them to bonor the contract they entered into is manifestly right and just. [Un contrato escrito que defina los derechos y obligaciones de las partes parece una forma apropiada de celebrar acuerdos de maternidad subrogada. Si las partes entienden sus derechos contractuales, solicitarles que honren el contrato que ellos celebraron es claramente correcto y justo] ${ }^{29}$.

No obstante lo anterior, existen también estados de la unión americana que le niegan validez a estos por considerarlos violatorios del orden público. En esta dirección, la legislación del estado de Louisiana estableció de manera clara y expresa que: A contract for surrogate motherbood as defined herein shall be absolutely null and shall be void and unenforceable as contrary to public policy. [Un contrato de maternidad subrogada como está definido allí será absolutamente inválido y será nulo y no exigible por ser contrario al orden público] ${ }^{30}$.

\footnotetext{
${ }^{28}$ Proyecto de Ley 037 de 2009, art. 5; Proyecto de Ley 196 de 2008, art. 5.

29 J.F. v. D.B., 116 Ohio St. 3d 363 (2007).

${ }^{30}$ Louisiana Code: $\$ 9: 2713$.
} 
Una postura similar se presenta en el estado de Washington en donde se considera que un contrato de maternidad subrogada es nulo por violar el orden público cuando este involucre el pago de una remuneración como contraprestación a su celebración:

\section{A surrogate parentage contract entered into for compensation, whether executed in the state of Washington or in another jurisdiction, shall be void and unenforceable in the state of Washington as contrary to public policy. [Un contrato de maternidad subrogada cele- brado por una compensación, sea ejecutado en el estado de Washington o en otra jurisdicción, será considerado nulo y no exigible en el estado de Washington por ser contrario al orden público]. En consecuencia, el contrato no es violatorio del orden público per se, sino únicamente cuando este involucre el pago de una remuneración en favor de la madre subrogada.}

Desde la perspectiva de la jurisprudencia, se ha considerado que los contratos de maternidad subrogada atentan contra el orden público por tres razones principales: a) constituyen un instrumento para la explotación de las mujeres, especialmente aquellas de bajos recursos económicos ${ }^{31}$, b) tienen como propósito la compraventa de recién nacidos, lo que convierte a estos en una mercancía que puede ser vendida y comprada como cualquier otro producto y de acuerdo con el precio del mercado ${ }^{32}$, y c) atentan contra la unidad de la familia, pues al intercambiarse un niño por una compensación, se está contribuyendo a la destrucción de una de las relaciones más importantes de la vida humana ${ }^{33}$.

${ }^{31}$ Generalmente una madre subrogada contará con menos recursos económicos que la pareja que busca ser padres mediante la celebración del contrato, situación esta que puede llevar a la existencia de un poder de negociación desproporcionado. En esta dirección se pronunció la Corte Suprema del estado de Nueva Jersey, en el caso In the Matter of Baby M, quien afirmó que en los contratos de maternidad subrogada, probablemente serán las personas con suficientes recursos económicos quienes tengan la calidad de padres adoptantes o interesados, mientras que las mujeres con una escasa capacidad económica serán quienes asuman la posición de madres subrogadas. Señaló entonces la corte: Nevertheless, it is clear to us that it is unlikely that surrogate mothers will be as proportionately numerous among those women in the top twenty percent income bracket as among those in the bottom twenty percent. Ibíd. Put differently, we doubt that infertile couples in the low-income bracket will find upper income surrogates. [Sin embargo, es claro para nosotros que es improbable que las madres subrogadas sean proporcionalmente tan numerosas entre aquellas mujeres ubicadas en el primer veinte por ciento del grupo de ingresos como entre las mujeres ubicadas en el último veinte por ciento del grupo de ingresos. Ibíd. En otras palabras, nosotros dudamos que las parejas infértiles en la parte baja del grupo de ingreso encuentren madres subrogadas del grupo superior]. In the Matter of Baby M, 109 N.J. 396 (1988). Igualmente, en el caso R.R. v. M.H., la corte reconoció que compensated surrogacy arrangements raise the concern that, under financial pressure, a woman will permit her body to be used and ber child to be given away. [Acuerdos de maternidad subrogada compensados generan la preocupación de que, bajo presiones económicas, una mujer permitirá que su cuerpo sea usado y su hijo tomado]. Véase también Cohen, B. Op. cit., p. 254.

32 Ferguson, S. A., "Surrogacy Contracts in the 1990's: The Controversy and Debate Continues”, en Duquesne Law Review, Duquesne Univerity, Vol. 4, Núm. 33, 1995, p. 906; Lascarides, D. E., “A Plea for the Enforceability of Gestational Surrogacy Contracts”, en Hofstra Law Review, Hofstra University, Vol. 4, Núm. 35, 1997, pp. 1240 y ss.

${ }^{33}$ Es así como, en el caso, In the Matter of Baby M, la corte señaló que el contrato de madre subrogada violaba el orden público toda vez que producía la separación permanente del recién nacido de uno de sus 
Sin embargo, estos argumentos no han sido aceptados de manera unánime. Por ejemplo, la Suprema Corte de California en el caso Johnson v. Calvert señaló que, aunque era razonable pensar que las mujeres de escasos recursos eran más propensas a celebrar contratos de maternidad subrogada ocupando en ellos la posición de madre subrogada, no existía prueba de que estos acuerdos explotaran a las mujeres pobres en mayor medida de lo que lo hacen los trabajos de bajos salarios o no deseables que estas deben aceptar como consecuencia de su necesidad económica. Adicionalmente, la corte indicó que no existía evidencia que sustentara la posición de que los contratos de maternidad subrogada promovieran el tratamiento de los recién nacidos como mercancías ${ }^{34}$.

Por otra parte, quienes defienden la validez de los contratos de maternidad subrogada, sostienen que estos no pueden ser invalidados, toda vez que la remuneración que se paga a la madre que presta su vientre para la gestación del feto, corresponde a una contraprestación por ese servicio, y no al del pago de un precio por la compra de un recién nacido ${ }^{35}$. Por lo tanto, el contrato de maternidad subrogada no involucra la realización de un contrato de compraventa de un bebé, sino el de uno de prestación de servicios.

\subsection{La validez del contrato de maternidad subrogada se determina también a partir de las normas del derecho de familia}

Otro rasgo distintivo que identifica la experiencia norteamericana frente a los contratos de maternidad subrogada es la utilización de normas del derecho de familia, particularmente las de adopción, para la determinación de la validez del contrato. En efecto, un segundo gran argumento que ha sustentado la posición negativa frente a los contratos de maternidad subrogada ha sido el considerar que estos violan las normas sobre adopción. Estas últimas son aplicadas a los contratos de maternidad subrogada,

padres naturales. Esta situación por lo tanto estaba en contravía con la política y el orden público del estado, el cual tiene como propósito que el bebé permanezca y crezca con ambos padres naturales en la mayor medida posible. In the Matter of Baby M, 109 N.J. 396 (1988).

${ }^{34}$ Although common sense suggests that women of lesser means serve as surrogate mothers more often than do wealthy women, there has been no proof that surrogacy contracts exploit poor women to any greater degree than economic necessity in general exploits them by inducing them to accept lower-paid or otherwise undesirable employment. We are likewise unpersuaded by the claim that surrogacy will foster the attitude that children are mere commodities; no evidence is offered to support it. The limited data available seem to reflect an absence of significant adverse effects of surrogacy on all participants. [Aunque el sentido común sugiere que las mujeres de menores recursos sirven como madres subrogadas de manera más frecuente que lo que lo hacen mujeres adineradas, no existe prueba de que los contratos de maternidad subrogada exploten a las mujeres en mayor grado de lo que la necesidad económica en general las explota por medio de inducirlas a aceptar salarios bajos o de otra forma trabajos no deseados. Nosotros igualmente no estamos convencidos por el argumento de que la subrogación promoverá la actitud de que los recién nacidos son meras mercaderías; no se presenta evidencia que lo sustente. La limitada información disponible parece reflejar una ausencia de efectos adversos significativos en todos los partícipes de la subrogación]. Johnson v. Calvert, 5 Cal. 4th 84 (1993).

${ }^{35}$ Cohen, B. Op. cit., p. 250. 
toda vez que el recién nacido que resulta del proceso de subrogación será adoptado por la esposa del padre biológico ${ }^{36}$.

La norma sobre adopción que se considera infringida, y que por tanto no permite calificar como válido un contrato de maternidad subrogada, es aquella que prohíbe el pago de una remuneración en una adopción de carácter privado. En efecto, se ha entendido que la remuneración que recibe la madre subrogada como consecuencia de la celebración del contrato es un pago que hace el padre biológico del recién nacido y su esposa para obtener la adopción del bebé, y no una remuneración a la madre subrogada por el servicio prestado. Aunque se alega que el pago que se hace a la madre subrogada tiene como propósito compensarla por permitir ser inseminada, prestar su cuerpo para el embarazo y padecer los efectos de este, la verdadera y principal intención del padre biológico y su esposa con el pago es que la madre les entregue la custodia del recién nacido. Por lo tanto, lo que realmente se busca con el contrato y la compensación económica a la madre biológica es la renuncia de esta a los derechos que como madre tiene sobre el recién nacido ${ }^{37}$.

En el caso In the Matter of Baby M, la Corte Suprema del estado de Nueva Jersey tuvo la oportunidad de decidir acerca de la validez de un contrato de maternidad subrogada. El contrato celebrado entre las partes establecía que el dinero pagado a la madre que prestaba su vientre para la gestación del bebé era una compensación por dicho servicio y los gastos derivados del contrato. Igualmente, el mismo contrato señalaba

${ }^{36}$ Ferguson, S. A., Op. cit., pp. 908-909. Sin embargo, la jurisprudencia de algunos estados ha rechazado la aplicación de las normas de adopción a los contratos de subrogación. En el caso Johnson v. Calvert, la Suprema Corte de California afirmó: Gestational surrogacy differs in crucial respects from adoption and so is not subject to the adoption statutes. [La subrogación gestacional se diferencia en aspectos cruciales de la adopción y por lo tanto no está sometida a las leyes de adopción].

37 One of the surrogacy contract's basic purposes, to achieve the adoption of a child through private placement, though permitted in New Jersey "is very much disfavored". Sees v. Baber, 74 N.J. 201, 217 (1977). Its use of money for this purpose -and we have no doubt whatsoever that the money is being paid to obtain an adoption and not, as the Sterns argue, for the personal services of Mary Beth Whitehead-is illegal and perhaps criminal. N.J.S. A. 9:3-54. In addition to the inducement of money, there is the coercion of contract: the natural mother's irrevocable agreement, prior to birth, even prior to conception, to surrender the child to the adoptive couple. Such an agreement is totally unenforceable in private placement adoption. Sees, 74 N.J. at 212-14. Even where the adoption is through an approved agency, the formal agreement to surrender occurs only after birth (as we read N.J.S.A. 9:2-16 and-17, and similar statutes), and then, by regulation, only after the birth mother has been offered counseling. [Uno de los propósitos fundamentales de los contratos de maternidad subrogada es alcanzar la adopción de un recién nacido mediante la colocación privada, aunque está permitida en Nueva Jersey "es desaprobada”. Sees v. Baber, 74 N.J. 201, 217 (1977). Su uso del dinero para este propósito -y no tenemos duda alguna de que el dinero está siendo pagado para obtener la adopción, y no como los Sterns lo argumentan, por los servicios personales de Mary Beth Whitehead - es ilegal y tal vez criminal. N.J.S.A. 9:3-54. Además del incentivo del dinero está la coerción del contrato: el acuerdo irrevocable de la madre natural, previo al nacimiento, aun previo a la concepción, para entregar el recién nacido a la pareja adoptante. Tal acuerdo es totalmente inválido en la adopción privada. Vea, 74 N.J. at 212-14. Aun donde la adopción se realiza por medio de una agencia aprobada, los acuerdos formales de entrega solamente ocurren después del nacimiento (como se lee en N.J.S.A. 9:2-16 y -17, and leyes similares), y luego, por ley, solamente después de que a la madre biológica le ha sido ofrecida asistencia].In the Matter of Baby M, 109 N.J. 396 (1988). 
expresamente que la remuneración por ningún motivo correspondía a un pago en favor de la madre como contraprestación a la terminación de sus derechos de custodia sobre el recién nacido, o por su consentimiento de entregarlo en adopción.

No obstante lo anterior, la corte calificó el pago como una compensación encaminada a obtener la adopción del menor. Dos razones principales le permitieron arribar a esta conclusión. Primero, el propio contrato establecía que el pago de la remuneración (\$10.000 dólares) ocurriría solamente cuando la madre subrogada hubiera renunciado a la custodia del niño y completado las obligaciones derivadas del contrato. Segundo, el acuerdo también indicaba que no se pagaría nada a la madre subrogada en caso de que el niño muriera antes del cuarto mes de embarazo, y que solamente pagaría $\$ 1.000$ dólares si el niño nacía muerto.

El argumento de considerar el contrato de maternidad subrogada inválido por considerarse que viola la prohibición de pagar una remuneración para la obtención de una adopción, podría también esgrimirse en el caso colombiano, toda vez que el artículo 74 de la Ley No 1098 de 2006 prohíbe claramente el pago de cualquier tipo de retribución por la entrega en adopción de un niño, niña o adolescente ${ }^{38}$.

Un argumento adicional que ha sustentado el rechazo a la validez de los contratos de maternidad subrogada con fundamento en las normas del derecho de familia, ha sido el considerar que estos constituyen un mecanismo para evitar la aplicación de las normas sobre custodia. En este sentido, la jurisprudencia se ha manifestado en los siguientes términos:

The provision in the surrogacy contract, agreed to before conception, requiring the natural mother to surrender custody of the child without any right of revocation is one more indication of the essential nature of this transaction: the creation of a contractual system of termination and adoption designed to circumvent our statutes. [La provisión en el contrato de maternidad subrogada, acordada antes de la concepción, requiriendo a la madre natural renunciar a la custodia del niño sin ningún derecho de revocación es una indicación más de la esencia natural de esta transacción: la creación de un sistema contractual de terminación y adopción diseñado para eludir nuestras leyes] ${ }^{39}$.

Desde estos lineamientos, no es posible reconocer como válidos los contratos de maternidad subrogada, pues no debe perderse de vista que el padre y la madre de un

38 “Ni el Instituto Colombiano de Bienestar Familiar ni las instituciones autorizadas por este para desarrollar el programa de adopción, podrán cobrar directa o indirectamente retribución alguna por la entrega de un niño, niña o adolescente para ser adoptado. En ningún caso podrá darse recompensa a los padres por la entrega que hagan de sus hijos para ser dados en adopción ni ejercer sobre ellos presión alguna para obtener su consentimiento. Tampoco podrán recibir donaciones de familias adoptantes previamente a la adopción. Quedan absolutamente prohibidas las donaciones de personas naturales o instituciones extranjeras a las instituciones colombianas como retribución por la entrega de niños, niñas o adolescentes en adopción”. Ley $\mathrm{N}^{\circ} 1098$ de 2006, art. 74.

${ }^{39}$ Ibid. 
recién nacido no pueden decidir sobre los derechos de custodia sobre él por un acuerdo privado. Lo anterior en razón a que la custodia de un menor debe estar sometida a un control judicial que tome en cuenta el mejor interés para él ${ }^{40}$. Esta posición fue adoptada en el caso Hawkins v. Frye, en donde la corte de familia decretó la invalidez del contrato de maternidad subrogada por considerar que la terminación de los derechos de custodia debía establecerse únicamente considerando el mejor interés del niño, y no mediante un contrato ${ }^{41}$. En la misma dirección, la Corte de Apelaciones del estado de Michigan afirmó: Surrogacy arrangements focus exclusively on the parents' desires and interests, and, accordingly, the parties are apt to be insensitive to what would be in the children's best interests. That position is in direct opposition to the child custody law in this state. [Los contratos de maternidad subrogada se centran exclusivamente en los deseos e intereses de los padres, y en consecuencia, las partes son insensibles a lo que serían los mejores intereses del niño] $]^{42}$. Igualmente, también se ha señalado que no tendría sentido que el legislador hubiera establecido restricciones para la terminación de los derechos de custodia, si fuera posible alcanzar ese mismo objetivo vía contractual ${ }^{43}$.

En el caso colombiano, sería posible también rechazar la validez de los contratos de maternidad subrogada bajo un argumento como el expuesto. En efecto, en Colombia la jurisprudencia constitucional ha reconocido que la figura de la patria potestad es de orden público, irrenunciable, intransferible e indisponible. En consecuencia, su ejercicio no es susceptible de ser "atribuido, modificado, regulado ni extinguido por la propia voluntad privada, sino en los casos que la propia ley lo permita" ${ }^{44}$. Adicionalmente, es el juez de familia quien tiene la facultad de decretar la suspensión o terminación de la patria potestad ${ }^{45}$. Por lo tanto, los particulares no podrían, vía contractual, establecer la titularidad de la patria potestad sobre un recién nacido.

Finalmente, en contra de la validez de los contratos de maternidad subrogada, se aduce también que estos no pueden considerarse válidos toda vez que los sentimientos de la madre biológica hacia el feto pueden cambiar durante el período del embarazo. En apoyo de este argumento, deben tomarse en cuenta las leyes de adopción que permiten un período de reflexión a la madre biológica para que, si así lo decide, pueda cambiar su opinión de entregar al niño y por tanto mantenerlo ${ }^{46}$. Por lo anterior, la jurisprudencia del estado de Massachusetts señaló que el consentimiento otorgado por la madre subrogada para renunciar a la custodia del recién nacido no era válido sino únicamente a partir del cuarto día transcurrido desde la fecha de nacimiento de este, término que resultaba de la aplicación analógica de las normas de adopción ${ }^{47}$. Consecuentemente, la renuncia

\footnotetext{
${ }^{40}$ R.R. v. M.H, 426 Mass. 501 (1998).

${ }^{41}$ Hawkins v. Frye, 1988 Del. Fam. Ct. LEXIS 31 (1988).

42 Doe v. Attorney General, 194 Mich. App. 432 (1992).

${ }^{43}$ In the Matter of Baby M, 109 N.J. 396 (1988).

${ }^{44}$ Corte Constitucional Colombiana, Sentencia C-145/10.

${ }^{45}$ Ley 1564 de 2012, art. 22.

${ }^{46}$ Ferguson, S. A., Op. cit., p. 912.

47 R.R. vs. M.H, 426 Mass. 501 (1998).
} 
irrevocable de la madre a los derechos de custodia sobre el niño hecha de manera previa al nacimiento no es válida, pues esta solamente lo será después del momento del parto y luego de haberle sido ofrecida asesoría profesional ${ }^{48}$.

Desde la perspectiva del derecho colombiano, este argumento también podría encontrar sustento, pues, al igual de lo que ocurre en los Estados Unidos, la ley colombiana en materia de adopción señala que "No tendrá validez el consentimiento que se otorgue para la adopción del hijo que está por nacer...Quien o quienes expresan su consentimiento para la adopción podrá revocarlo dentro del mes siguiente a su otorgamiento” ${ }^{49}$. Estas normas constituyen un reconocimiento de la posibilidad, de que luego del parto, los sentimientos de la madre puedan cambiar, y en consecuencia, así también su decisión de entregar al recién nacido ${ }^{50}$.

\subsection{Las leyes que regulan la celebración de los contratos de maternidad subrogada buscan contrarrestar los efectos negativos derivados del contrato}

Finalmente, la experiencia norteamericana en materia de contratos de maternidad subrogada se caracteriza porque la regulación particular aplicable a estos está encaminada a contrarrestar los efectos negativos que de ellos se derivan. En efecto, como fue mencionado en líneas anteriores, algunos estados de la unión americana promulgaron leyes especiales dirigidas a regular de manera profunda el mencionado contrato. Aunque el contenido establecido por estas legislaciones estatales no es de carácter uniforme, de la lectura, estudio y análisis de las mismas es posible identificar elementos comunes, entre los cuales se destacan los siguientes:

1) Debe existir una aprobación judicial del contrato. La revisión judicial tiene como propósito que el juez verifique que el padre biológico y su esposa tienen la capacidad o aptitud necesaria para adoptar, que las partes celebraron voluntariamente el contrato de subrogación y que entienden sus términos, naturaleza, significado y el efecto de su ejecución.

Para el caso colombiano, los proyectos de ley estudiados no señalaron un requisito similar. Sin embargo, sería importante su establecimiento, pues de esta manera podría

\footnotetext{
${ }^{48}$ In the Matter of Baby M, 109 N.J. 396 (1988).

49 Art. 66, Ley 1098 de 2006.
}

${ }^{50}$ La doctrina colombiana ha señalado frente a esta norma lo siguiente: "Los progenitores, pero especialmente la madre embarazada en circunstancias personales no ordinarias, tiene unos sentimientos encontrados respecto de la criatura que se encuentra en el vientre y entran en conflicto la visión nacional de la situación social, la carga que tendrán que asumir con la natural limitación de la libertad y el manejo de responsabilidades, frente al instinto de reproducción de la especie; de modo que es posible que en estos momentos puedan considerar preferible desprenderse de su hijo para darlo en adopción; pero está documentado científicamente que cuando llega la hora del parto, la situación se invierte y en ese momento la fuerza instintiva prima sobre la racional y ya no se ve tan acertada su resolución de entregar su hijo, especialmente cuando se supera esa conocida etapa de la depresión posparto, debido a los cambios hormonales de pasar del embarazo a la maternidad”. Medina, J. E., Derecho Civil. Derecho de Familia, $2^{a}$ edición, Editorial Universidad del Rosario, 2010, p. 479. 
verificarse el cumplimiento de los requisitos señalados en la ley antes de la realización del procedimiento médico. De esta manera, podría asegurarse que los contratos de maternidad subrogada solo se realicen en situaciones excepcionales, como lo es una situación de infertilidad.

2) Las partes del contrato, necesariamente mayores de edad, deben otorgar su consentimiento por escrito autorizando la ejecución del procedimiento médico de inseminación.

En el caso colombiano, se exigió también en los proyectos de ley que las partes del contrato no solo dieran su consentimiento por escrito, sino que también fueran mayores de edad. Sin embargo, frente a esto último, el requisito pareciera más exigente, pues la edad mínima para los padres solicitantes y la madre subrogada se estableció en 25 $a_{n}{ }^{51}$. Vale la pena resaltar que esta es la misma edad exigida por la ley colombiana en materia de adopción ${ }^{52}$.

3) Solamente podrán tener la calidad de madres subrogadas aquellas mujeres que puedan demostrar documentalmente que ya han dado a luz anteriormente en al menos una oportunidad.

Los proyectos de ley estudiados para el caso colombiano tampoco establecieron este requisito. Sin embargo, su fijación podría considerarse importante en la construcción de una regulación colombiana sobre la materia, pues así lograría garantizarse que la madre gestante ya hubiese experimentado previamente los efectos físicos y psicológicos que envuelve un embarazo y el nacimiento de un bebé. De esta manera, su consentimiento para ser parte de un contrato de maternidad subrogada será mucho más completo e informado.

4) Es obligatoria la realización de una evaluación médica a la madre subrogada. Esta tiene como propósito no solo demostrar que la madre tiene la capacidad médica para someterse al procedimiento quirúrgico, sino también que tiene las condiciones fisiológicas para dar a luz, sin que ello implique un riesgo para su salud o la del recién nacido.

Un requisito similar se estableció en los proyectos de ley presentados al Congreso colombiano. Por ejemplo, los proyectos de Ley 037 de 2009 y 196 de 2008 establecieron en su artículo $6^{\circ}$, como requisito para adelantar el procedimiento de gestación sustitutiva, la realización de todos los exámenes necesarios a la madre gestante sustitutiva.

5) Adicionalmente, todas las partes del contrato deben someterse a una evaluación sicológica realizada por un siquiatra, sicólogo, consejero pastoral, o trabajador social. El evaluador deberá mantener un registro de los resultados y conclusiones de la evaluación y entregar una copia de los mismos a las partes del contrato. Igualmente, estas últimas deben entregar a sus contrapartes la copia de los resultados de su evaluación. La realización de este examen sicológico tiene como objetivo verificar, por una parte, la capacidad de la persona evaluada para dar amor, afecto y guía al bebé, y por el otro, su capacidad para asumir los riesgos inherentes al contrato de maternidad subrogada.

51 Proyecto de Ley 037 de 2009, art. 3. Proyecto de Ley 196 de 2008,

52 "Requisitos para adoptar. Podrá adoptar quien, siendo capaz, haya cumplido 25 años de edad, tenga al menos 15 años más que el adoptable, y garantice idoneidad física, mental, moral y social suficiente para suministrar una familia adecuada y estable al niño, niña o adolescente. Estas mismas calidades se exigirán a quienes adopten conjuntamente...”.. Ley 1098 de 2006, art. 68. 
En el caso colombiano, la realización de una evaluación de tipo sicológico a las partes involucradas aparece también como un requisito necesario. En efecto, el proyecto de ley 037 de 2009 señalaba en su artículo $3^{\circ}$, la necesidad de que la pareja solicitante del procedimiento estuviera en condiciones psicológicas aptas, y que la madre gestante sustitutiva se hubiese practicado un examen psicológico. Adicionalmente, el artículo $8^{\circ}$ del mencionado proyecto establecía la obligación de adelantar un acompañamiento psicológico a todas las partes del contrato, con el propósito de evitar la ocurrencia de consecuencias psicológicas negativas a la criatura y a la madre gestante. Idénticos requisitos se establecieron en el Proyecto de Ley No 196 de 2008.

6) La agencia gubernamental competente o una agencia de adopción autorizada debe realizar una visita al hogar de los contratantes. El propósito de esta visita es valorar la capacidad y disposición de las partes para proveer al recién nacido con comida, vestido, techo, cuidado médico y, en general, sus necesidades básicas.

En el caso colombiano, los proyectos de ley estudiados no señalaron un requisito similar. Sin embargo, su establecimiento podría considerarse como un aspecto positivo en aras de garantizar que el recién nacido crezca dentro de un contexto familiar adecuado para su normal desarrollo y crianza.

7) El contrato no puede establecer el pago de una compensación a favor de la madre subrogada. Si ello es así, el acuerdo se considerará nulo. Sin embargo, en caso de que la madre subrogada reciba una compensación, la cláusula de pago estará limitada a cubrir los gastos médicos relacionados con el embarazo, la elaboración de evaluaciones médicas y no médicas, la pérdida de los salarios de la madre cuando la ausencia del trabajo sea recomendada por escrito por un médico, la celebración de un contrato de seguro de salud, incapacidad y vida durante el término del embarazo y hasta seis semanas después, y los gastos legales razonables que se deriven del contrato.

Para el caso colombiano, las propuestas de regulación colombiana al contrato de maternidad subrogada, establecieron también el carácter gratuito del contrato celebrado entre los padres solicitantes y la madre subrogada. En efecto, el carácter gratuito del contrato de madre subrogada se desprende de la definición que los proyectos de Ley 037 de 2009 y 196 de 2008 establecieron: "acuerdo gratuito, formal y confidencial, realizado entre la receptora gestante sustitutiva y la pareja solicitante con el fin de llevar a feliz término el proceso de gestación".

No obstante lo anterior, el carácter gratuito del contrato parece verse limitado por el artículo 15 de los mismos proyectos, el cual impone como obligaciones a la pareja interesada: "5. Cubrir los gastos médicos de la receptora gestante sustitutiva durante el período de gestación. 6. Cubrir los exámenes solicitados por el especialista durante el período de gestación. 7. Cubrir los gastos de alimentación de la receptora gestante sustitutiva. 8. Cubrir los gastos de vestido de la receptora gestante sustitutiva. 9. Entregar un auxilio económico mensual a la mujer receptora gestante sustitutiva para su sostenimiento".

Desde este contexto, es posible identificar una gran similitud existente entre la regulación norteamericana y la colombiana, pues en ambas se rechaza el pago de una compensación a la madre gestante como contraprestación por la celebración del 
contrato. Los únicos pagos que estarían permitidos serían aquellos que se generan como consecuencia de la situación de embarazo de la madre gestante, pero que en ningún caso están encaminados a compensarla o retribuirle económicamente la decisión de celebrar el contrato.

8) El contrato de maternidad subrogada debe incluir además una manifestación de cada una de las partes, indicando que ellas han leído y entendido el contrato, que conocen y entienden sus derechos y responsabilidades y que fue celebrado de manera voluntaria y con pleno conocimiento de su contenido.

Para el caso colombiano, una exigencia similar se identifica en los proyectos de ley 037 de 2009 y 196 de 2008, los cuales señalaron que debe existir un consentimiento voluntario, expreso, libre de vicios y por escrito para la realización del procedimiento médico.

9) El acuerdo debe también contener una cláusula que exprese el consentimiento de la madre subrogada de renunciar a la custodia del recién nacido o que acepta la obligación de custodia si ella decide mantener sus derechos sobre este. Igualmente debe aparecer el de su esposo, en caso de que la madre se encuentre casada. En el caso del padre biológico y su esposa, estos deben manifestar su aceptación de las obligaciones de custodia sobre el recién nacido.

Los proyectos de ley 196 de 2008 y 37 de 2009 establecieron una situación similar, pues, por un lado, imponían como obligaciones para la pareja solicitante las de recibir y no rechazar a la criatura una vez que esta naciera y, por el otro, le señalaban a la madre gestante la obligación de entregar al recién nacido y renunciar a la filiación sobre este.

10) Finalmente, la regulación norteamericana reconoce la posibilidad a la madre subrogada de manifestar su intención de mantener al recién nacido dentro de las 72 horas siguientes al nacimiento, excepto que circunstancias extraordinarias no le hayan permitido tomar una decisión, en cuyo caso el período será de una semana. Si la madre decide ejercer su derecho a mantener la custodia sobre el niño, deberá informar por escrito de su intención a las otras partes del contrato y al médico o al director del hospital.

Este requisito no aparece establecido en los proyectos de ley estudiados. Sin embargo, su incorporación podría ser importante con el propósito de proteger a la madre gestante en la eventualidad de que decida cambiar su opinión, tal como se reconoce para la figura de la adopción en el artículo 66 de la Ley Nº 1098 de 2006.

La lectura de los requisitos y procedimientos adoptados por las diferentes leyes de los estados de la unión americana, hace posible identificar un interés del legislador de permitir la celebración de los contratos de maternidad subrogada a partir de mecanismos que, por un lado, contrarresten los efectos negativos derivados de la celebración del contrato y, por el otro, protejan a la madre subrogada.

En efecto, tal como se manifestó anteriormente, los contratos de maternidad subrogada han sido criticados y rechazados al considerarse que: a) envuelven el riesgo de que la madre subrogada sufra daños o secuelas de tipo físico o sicológico, b) desconocen el derecho de la madre subrogada a arrepentirse de renunciar a los derechos de custodia sobre el recién nacido, c) son instrumentos para la explotación femenina, y d) promueven la venta de recién nacidos. 
Al revisar las características principales de las normas estatales, se encuentra que las críticas que recaen sobre el contrato de maternidad subrogada buscan ser superadas por varios mecanismos. Con relación a los efectos físicos y sicológicos que puede enfrentar la madre subrogada, las normas imponen la obligación de que las partes del contrato se sometan a evaluaciones médicas con profesionales debidamente acreditados. Igualmente se destaca como requisito obligatorio que la madre subrogada ya haya dado a luz con anterioridad. Claramente estos requisitos buscan garantizar que las partes del contrato cuenten con las capacidades físicas y sicológicas para asumir las consecuencias derivadas del acuerdo de voluntades.

Adicionalmente, se reconoce a la madre subrogada la posibilidad de cambiar su decisión de renunciar a los derechos de custodia sobre el menor, hasta un momento después del nacimiento, siguiendo el mismo principio establecido para los casos de adopción tradicionales.

También se destaca la prohibición de que la madre subrogada reciba una compensación como consecuencia de la celebración del contrato. Esta restricción puede interpretarse como un mecanismo encaminado a evitar el abuso de mujeres que se encuentran en situaciones de dificultad económica, y que en consecuencia puedan verse forzadas a servir como madres subrogadas ${ }^{53}$.

Finalmente, debe resaltarse que muchos de los requisitos establecidos por las leyes norteamericanas fueron reconocidos en los proyectos de ley colombianos, lo que demuestra un claro acercamiento entre las dos posturas estudiadas. De igual manera, es importante resaltar que aquellos aspectos no considerados en los proyectos colombianos deberían ser tomados en cuenta en el futuro, pues persiguen o tiene como objetivo la protección del recién nacido o la madre subrogada.

\section{CONCLUSIONES}

El análisis de la legislación y jurisprudencia estadounidense permite construir e identificar la experiencia del país norteamericano frente al contrato de maternidad subrogada. Esta experiencia tiene como rasgos distintivos los siguientes: a) existe una

53 La necesidad de evitar la explotación económica de la mujer mediante el contrato de maternidad subrogada ha sido también reconocida por la jurisprudencia. En el caso R.R. v. M.H., la corte de Massachusetts señaló que los contratos de maternidad subrogada no son válidos cuando estos establecen el pago de una remuneración en favor de la madre subrogada. La justificación para dicha situación se encuentra en la necesidad de eliminar cualquier retribución de tipo económico en favor de esta con el propósito de garantizar que no exista una presión económica que la lleve a celebrar el contrato. En palabras de la corte compensated surrogacy arrangements raise the concern that, under financial pressure, a woman will permit her body to be used and her child to be given away. Sin embargo, la propia corte reconoció que un contrato de maternidad subrogada no es ilegal si el pago de la compensación en favor de la madre subrogada no tiene como objetivo remunerarla por su renuncia a los derechos de custodia sobre el recién nacido y adicionalmente se entiende que el consentimiento de la madre a renunciar a la custodia del bebé no la obliga sino hasta el vencimiento de un período adecuado y posterior al nacimiento. 
regulación del contrato a partir de la ley y la jurisprudencia, b) no hay una posición uniforme entre los estados frente a la validez jurídica del contrato, c) se acude a la noción de orden público y a las normas del derecho de familia para determinar su validez, y d) la regulación del contrato está encaminada a contrarrestar los efectos negativos que se derivan de este.

Desde este contexto, es posible identificar aspectos comunes entre la postura norteamericana y la visión colombiana, analizada o construida esta última a partir de la jurisprudencia de la Corte Constitucional y los diversos intentos legislativos presentados. En este sentido, se acepta la celebración de contratos de maternidad subrogada, aunque sometida al cumplimiento de ciertos requisitos encaminados a la protección del recién nacido y evitar la explotación de la madre gestante. Igualmente, frente a estos requisitos, aunque existen varios puntos en común, también se presentan algunas diferencias, especialmente por la ausencia de regulación en el caso colombiano.

Finalmente, no puede negarse que la práctica de la subrogación es una realidad, y por tanto el derecho, como herramienta reguladora de las acciones sociales, debe asumir una postura determinada frente a esta. En este sentido, la aproximación estadounidense al contrato de maternidad subrogada puede servir como fuente inspiradora para aquellos países que carecen de un tratamiento legislativo y jurisprudencial frente a esta materia, aunque claramente sin olvidar que serán las condiciones económicas, sociales y políticas de estos las que determinen su tratamiento.

\section{BIBLIOGRAFÍA}

Aвraham. Behm, L. L., "Legal, Moral \& International Perspectives on Surrogate Motherhood: the Call for a Uniform Regulatory Scheme in the United States", en DePaul Journal of Health Care Law, DePaul University, Vol. 3, Núm. 2, 1999.

Cohen, B., "Surrogate Mothers: Whose Baby Is It?", en American Journal of Law E Medicine, Boston University School of Law, Vol. 3, Núm. 101984.

Ferguson, S. A., "Surrogacy Contracts in the 1990's: The Controversy and Debate Continues", en Duquesne Law Review, Duquesne Univerity, Vol. 4, Núm. 33, 1995.

GalleE, C. L., "Surrogate Mother Contracts: A View of Recent Legislative Approaches", en Journal of Health Law, American Health Law Associations, Vol. 6, Núm. 24, 1992.

Gugucheva, M., Surrogacy in America, Council for Responsible Genetics, 2010, disponible en http://www.councilforresponsiblegenetics.org/pageDocuments/KAEVEJ0A1M.pdf (última visita 01/03/12).

Jadva, V., Murray, C., Lycett, E., Maccallum, F. y Golombok, S., "Surrogacy: the Experiences of Surrogate Mothers", en Human Reproduction, Oxford University Press, Vol. 10, Núm. 18, 2003.

Lascarides, D. E., "A Plea for the Enforceability of Gestational Surrogacy Contracts", en Hofstra Law Review, Hofstra University, Vol. 4, Núm. 35, 1997.

Marín Vélez, G. A., "El arrendamiento de vientre en Colombia", en Opinión Jurídica, Sello editorial Universidad de Medellín, Vol. 3, 2003.

Mclachlan, H. V. y Swales, J. K., "Show Me the Money: Making Markets in Forbidden Exchange: Commercial Surrogate Motherhood and the Alleged Commodification of 
Children: a Defense of Legally Enforceable Contracts", en Law and Contemporary Problems, Duke University, Vol. 3, Núm. 72, 2009.

Medina, J. E., Derecho Civil Derecho de Familia, $2^{a}$ edición, Editorial Universidad del Rosario, 2010.

Spivack, C., "The Law of Surrogate Motherhood in the United States", en The American Journal of Comparative Law, American Society of Comparative Law, Núm. 58, Suplemento, 2010.

Tiller, S. L., "Litigation, Legislation, and Limelight: Obstacles to Commercial Surrogate Mother Arrangements”, en Iowa Law Review, Iowa University, Vol. 2, Núm. 72, 1987.

Van Den Akker, O., "Psychosocial aspects of surrogate motherhood", en Human Reproduction Update, Oxford University Press, Vol. 1, Núm. 13, 2007.

\section{Jurisprudencia}

Cynthia v. Moschetta, 25 Cal. App. 4th 1218 (1994).

Doe v. Attorney General, 194 Mich. App. 432 (1992).

Hawkins v. Frye, 1988 Del. Fam. Ct. LEXIS 31 (1988).

In the Matter of Baby M, 109 N.J. 396 (1988).

J.F. v. D.B., 116 Ohio St. 3d 363 (2007).

Johnson v. Calvert, 5 Cal. 4th 84 (1993).

J.R. v. The State of Utah, 261 F. Supp. 2d 1268 (2003).

R.R. v. M.H, 426 Mass. 501 (1998).

Corte Constitucional, Sentencia C-145/10, M.P. Gabriel Eduardo Mendoza Martelo, 03/03/2010.

Corte Constitucional, Sentencia T-968/09, M.P. María Victoria Calle, 18/12/2009. 
